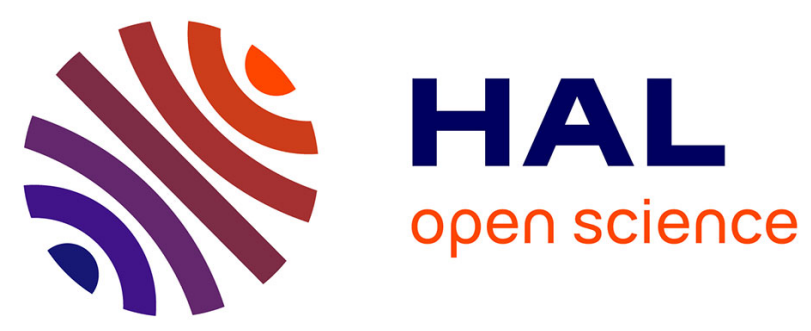

\title{
On the design of capacitive sensors using flexible electrodes for multipurpose measurements
}

Pierre Thibault, Pantxo Diribarne, Thierry Fournier, Sylvain Perraud, Laurent Puech, P.-Etienne Wolf, Bernard Rousset, Roser Vallcorba

\section{To cite this version:}

Pierre Thibault, Pantxo Diribarne, Thierry Fournier, Sylvain Perraud, Laurent Puech, et al.. On the design of capacitive sensors using flexible electrodes for multipurpose measurements. Review of Scientific Instruments, 2007, 78 (4), pp.043903. 10.1063/1.2721406 . hal-03047945

\section{HAL Id: hal-03047945 \\ https://hal.science/hal-03047945}

Submitted on 9 Dec 2020

HAL is a multi-disciplinary open access archive for the deposit and dissemination of scientific research documents, whether they are published or not. The documents may come from teaching and research institutions in France or abroad, or from public or private research centers.
L'archive ouverte pluridisciplinaire HAL, est destinée au dépôt et à la diffusion de documents scientifiques de niveau recherche, publiés ou non, émanant des établissements d'enseignement et de recherche français ou étrangers, des laboratoires publics ou privés. 


\section{AIP}

\section{On the design of capacitive sensors using flexible electrodes for multipurpose measurements}

Pierre Thibault, Pantxo Diribarne, Thierry Fournier, Sylvain Perraud, Laurent Puech et al.

Citation: Rev. Sci. Instrum. 78, 043903 (2007); doi: 10.1063/1.2721406

View online: http://dx.doi.org/10.1063/1.2721406

View Table of Contents: http://rsi.aip.org/resource/1/RSINAK/v78/i4

Published by the American Institute of Physics.

\section{Additional information on Rev. Sci. Instrum.}

Journal Homepage: http://rsi.aip.org

Journal Information: http://rsi.aip.org/about/about_the_journal

Top downloads: http://rsi.aip.org/features/most_downloaded

Information for Authors: http://rsi.aip.org/authors

\section{ADVERTISEMENT}
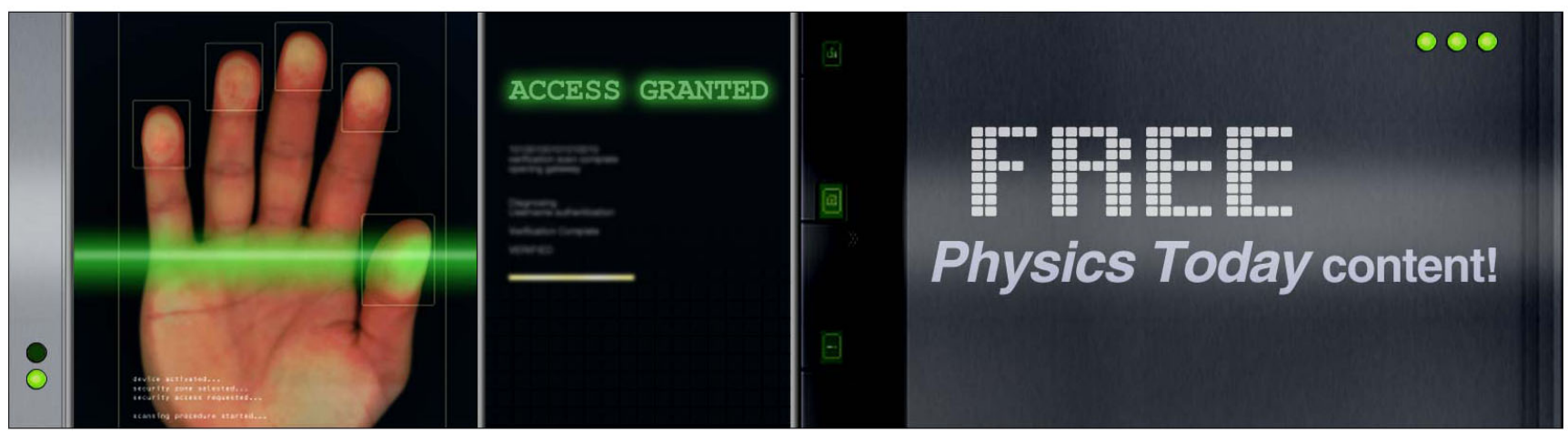


\title{
On the design of capacitive sensors using flexible electrodes for multipurpose measurements
}

\author{
Pierre Thibault, ${ }^{\text {a) }}$ Pantxo Diribarne, Thierry Fournier, Sylvain Perraud, \\ Laurent Puech, and P.-Etienne Wolf \\ Centre de Recherches sur les Très Basses Températures, laboratoire associé à l'Université Joseph Fourier, \\ CNRS, BP 166, 38042 Grenoble Cedex 9, France
}

Bernard Rousset and Roser Vallcorba

CEA-Grenoble/DRFMC/SBT, 17 rue des Martyrs, 38054 Grenoble Cedex 9, France

(Received 13 November 2006; accepted 9 March 2007; published online 11 April 2007)

\begin{abstract}
This article evaluates the potential of capacitive measurements using flexible electrodes to access various physical quantities. These electrodes are made of a thin metallic film, typical thickness $0.2 \mu \mathrm{m}$, evaporated on a plastic substrate. Their large flexibility enables them to be mounted in complex geometries such as curved surfaces. In the configuration of planar condensers, using a very sensitive commercial capacitive bridge and a three-terminal measurement method, several measurements are presented. A relative resolution of $10^{-8}$ for the thermal expansion of samples is obtained at low temperature in a differential configuration. The same technique adopted for pressure gauge measurements at low temperature led to a typical $0.1 \mathrm{~Pa}$ resolution over a dynamic range of $10^{4} \mathrm{~Pa}$. In the configuration of interleaved electrodes, condensers have been used to measure wetting by either bulk liquid helium or by thin continuous helium films in a cylindrical pipe. Both experimental and numerical evidence is provided, showing that the close proximity of a reference ground potential significantly increases the relative sensitivity to fluid wetting. Further, interleaved electrodes can be used to access both the area that is covered by a liquid film but also to determine the thickness of this film, provided it is comparable to the periodicity of the electrode pattern. (c) 2007 American Institute of Physics. [DOI: 10.1063/1.2721406]
\end{abstract}

\section{INTRODUCTION}

Capacitive sensors are widely used to measure various physical quantities. The review by Adams ${ }^{1}$ points out the main characteristics and possible designs in the case of pressure gauge measurements. However, in many situations of interest, it proves to be difficult to build a system that fully obeys the law of a parallel plate condenser with a capacitance given by $C=\varepsilon_{S}(S / l)$, where $\varepsilon_{S}=\varepsilon_{R} \times \varepsilon_{0}$, with $\varepsilon_{0}$ the dielectric constant in vacuum, $\varepsilon_{R}$ the relative permittivity of the medium separating the electrodes, $S$ their common surface, and $l$ the gap. To approach this limit, metallic electrodes deposited on a flexible plastic foil (like Kapton ${ }^{\mathrm{TM}}$ polyimide film) can be used, the plastic film being further glued on metallic supports attached to the measuring cell. If a guard ring is simultaneously evaporated, consisting of a metallic grounded region at a distance shorter than the length $l$ surrounding the active electrode, a good approximation of a parallel plate condenser is experimentally realized.

Such a design is described in Sec. II and successfully illustrated by thermal expansion measurements of samples of typical length $L=1 \mathrm{~cm}$ with a typical resolution $\Delta L / L$ $=10^{-8}$. In Sec. III, the same principle is applied to the construction of a pressure gauge for cryogenic use that is leaktight with a membrane on which the electrodes were glued. In Sec. IV, these techniques are generalized to liquid level

\footnotetext{
${ }^{a)}$ Electronic mail: pierre.thibault@ujf-grenoble.fr
}

measurements using interleaved electrodes. In this case, the dielectric constant and the thickness of the polyimide substrate come into play to determine the sensitivity. This issue is illustrated by a number of experiments and by numerical simulations, as this design may encounter a great deal of applications.

\section{THERMAL EXPANSION MEASURING CELL}

While designing a condenser for a specific use, a very pragmatic solution with moderate input consists of optimizing the ratio between the response to changes of the quantity of interest (let us say the length of a sample), to that due to the change of the environment, which should ideally be zero. In the situation of an ideal parallel plate condenser, considering two electrodes separated by a gap $l \approx 0.1 \mathrm{~mm}$, surface area $S \approx 1 \mathrm{~cm}^{2}$, the capacitance in vacuum is only $C$ $\approx 8.85 \mathrm{pF}$. The measurement of this capacitance with a Sauty-type bridge would already give a decreased sensitivity due to the serial combination of the coaxial lead capacitance and that of the sample. Typically, with standard coaxial lines, this can be $100 \mathrm{pF}$ per meter and the corresponding sensitivity is reduced by a decade or more. The use of capacitive bridges in the well-known three-terminal configuration as described in Ref. 1 is therefore advantageous, as it is not sensitive to the mutual capacitance between the electrodes and the ground. 


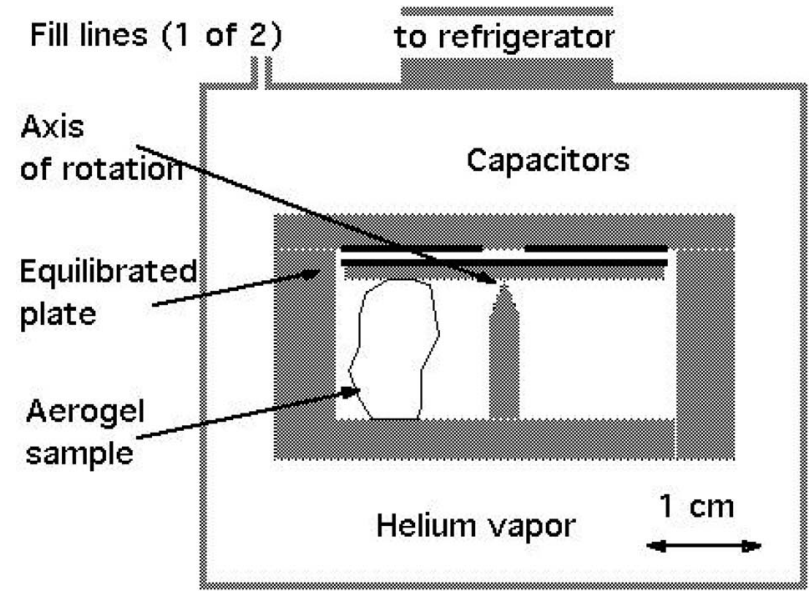

FIG. 1. Side view of a dilatometer based on a differential capacitive detection. Sample expansion is measured respective to that of the copper cell body. A common electrode is glued on the central moving part. Small copper pins serve for the axis of rotation, without significant friction.

According to this principle, the sensors described in this paper obey four rules.

(i) The two electrodes are electrically well-insulated from the ground, which also acts as an electrical shield.

(ii) The electrodes are the major source of the signal. This is obtained by keeping the capacitance large compared to that of the connecting leads.

(iii) The approximation of negligible finite-size effects is valid. The fact that the electrical field lines are not everywhere perpendicular to the surface of the electrodes is responsible for leakage of the field, which can be minimized if a grounded conductor (or guard ring) is placed around the electrode at a distance shorter than the gap.

(iv) Whenever possible, a differential geometry is preferred in order to enhance the sensitivity.

These points are illustrated in the cell geometry of Fig. 1, which was used for thermal expansion measurements of silica-aerogels at low temperature, shown in Fig. 2. (See Ref. 2 for the context of this experiment.) The electrodes were drawn on single-face copper-coated Kapton, and the guard ring obtained by chemical etching of the copper using a photosensitive resin. After gluing with epoxy on the copper cell body, they form two parallel plate condensers.

When the sample expands or contracts relatives to the copper cell, the gaps on both sides of the rotation axis, $l_{X}$ and $l_{R}$, change in opposite directions from their initial values. The measurements were performed using a differential bridge commercialized by Barras-Provence. ${ }^{3}$ While measuring the ratio between the unknown capacitance $C_{X}$ and the reference capacitance $C_{R}$, the dielectric constant of the fluid does not appear in the quantity $X^{-1}=1+C_{R} / C_{X}=1+l_{X} / l_{R}$. Furthermore, this configuration ensures that the force exerted on the sample under measurement is small and constant, as the Archimedes forces on plates compensate each other. This point is of importance as these samples have an unusually low bulk modulus (a few bars) and must be measured free of

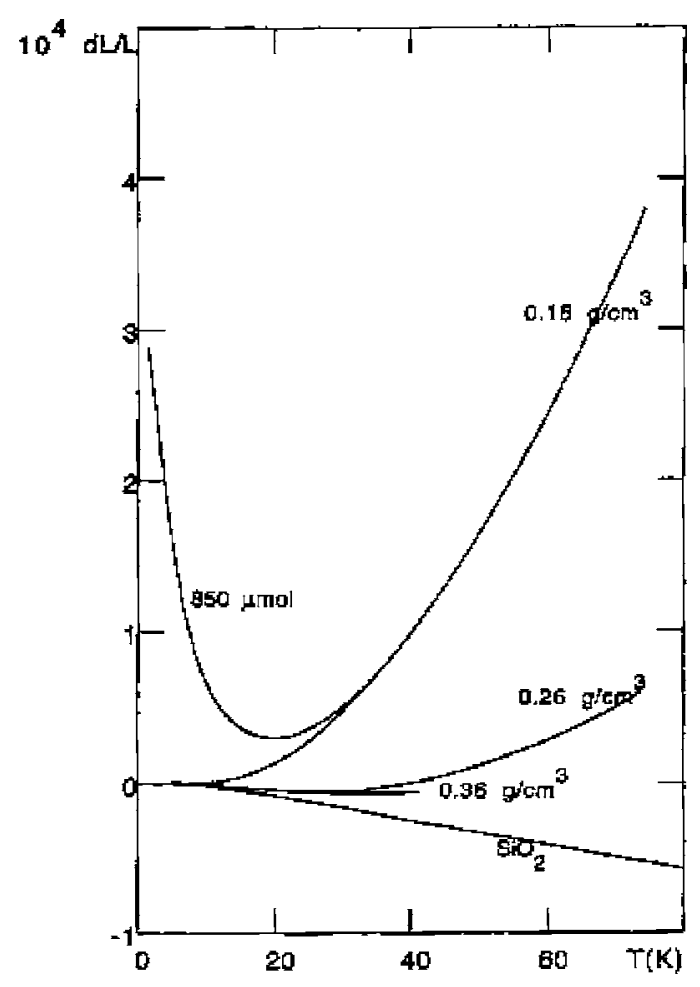

FIG. 2. Thermal expansion $\Delta L / L$ of some dry aerogels and of amorphous silica. The expansion of the aerogel of density $\rho=0.16 \mathrm{~g} \mathrm{~cm}^{-3}$ in the presence of gas introduced into the cell is also shown. The data reveal a large expansion due to the adsorption of helium which relaxes surface stress.

stress. The best relative resolution achieved in this configuration was $10^{-8}$, corresponding to the nominal resolution of the bridge.

\section{PRESSURE GAUGE}

A pressure gauge has also been designed according to specifications (i) to (iv). The whole mechanical system (excluding the electrodes) is depicted in Fig. 3. It relies on a capacitive measurement of the deflection of a $\mathrm{Cu}-\mathrm{Be}$ flexible membrane onto which the electrodes are glued. The membrane of thickness $e=0.15 \mu \mathrm{m}$ is rigidly held at the edges. The change in capacitance resulting from a deformation of the membrane is measured in a differential manner. Electrodes $1 \mathrm{~cm}$ in diameter are centered on the middle of the membrane and surrounded by a guard ring. The two halves of the copper cell are sealed onto either side of the $\mathrm{Cu}-\mathrm{Be}$ membrane using Helicoflex aluminum seals. The cell is made leak-tight at a level of $10^{-10} \mathrm{mbar} \times 1 / \mathrm{s}$.

A calibration at room temperature is given in Fig. 4. The sensibility is good over a large pressure range and the resolution excellent even over a reduced pressure range. For small deflections of the membrane, the deformation of the membrane relative to the gap $l_{0}$ is approximated by ${ }^{4}$

$$
\frac{z(r)}{l_{0}}=\frac{\Delta p}{Y} \frac{\left(R^{2}-r^{2}\right)^{2}}{l_{0} e^{3}} \frac{3}{16}\left(1-\nu^{2}\right),
$$

where $\Delta p$ is the pressure difference, $Y$ the Young modulus, $R$ the membrane radius, $r$ the electrode's radius, $e$ the thickness of the membrane, and $\nu$ the Poisson ratio. For larger deflections, the deflection of the membrane is not uniform, and 


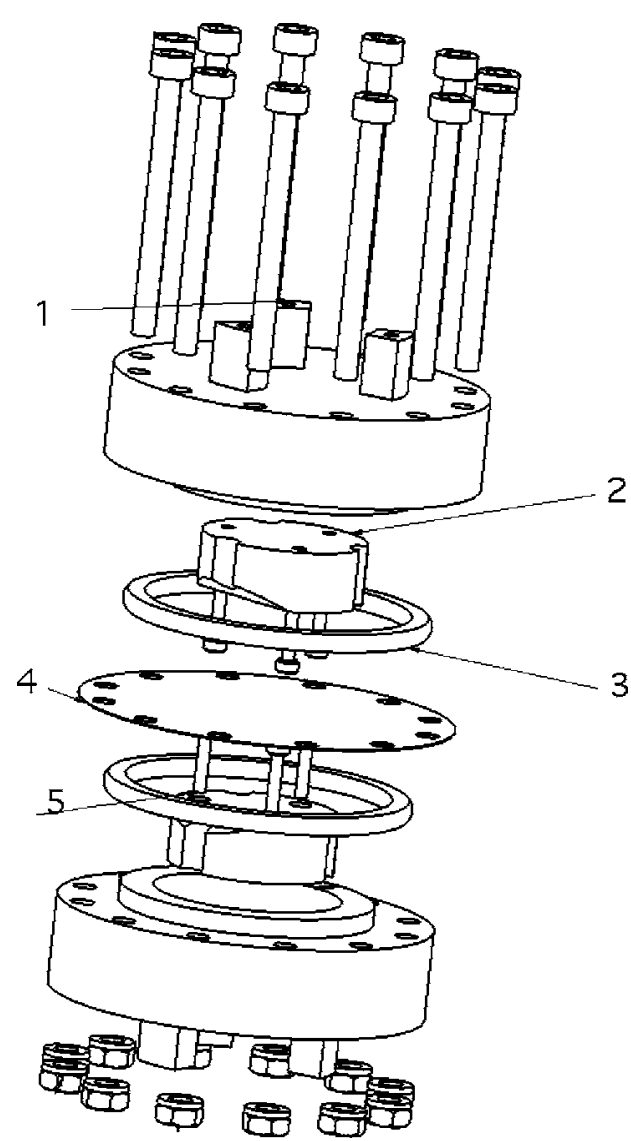

FIG. 3. Left: Expanded view of the pressure cell. (1) Fill line and electrical connections. (2) and (5) Support for the soft electrodes. (3) Helicoflex aluminum ring. (4) $\mathrm{Cu}-\mathrm{Be}$ membrane.

depends on the radial position. Rewriting Eq. (1) in dimensionless form $z(r) / l_{0}=\left(R^{2}-r^{2}\right)^{2} / a(p)^{4}$, an integration leads for each condenser to

$C_{L P}=-\pi \varepsilon_{0} \varepsilon_{R}^{L P} \frac{a^{2}}{l_{0}} \frac{1}{2}\left\{\ln \left(\frac{1+\frac{R^{2}-r^{2}}{a^{2}}}{1-\frac{R^{2}-r^{2}}{a^{2}}}\right)-\ln \left(\frac{1+\frac{R^{2}}{a^{2}}}{1-\frac{R^{2}}{a^{2}}}\right)\right\}$,

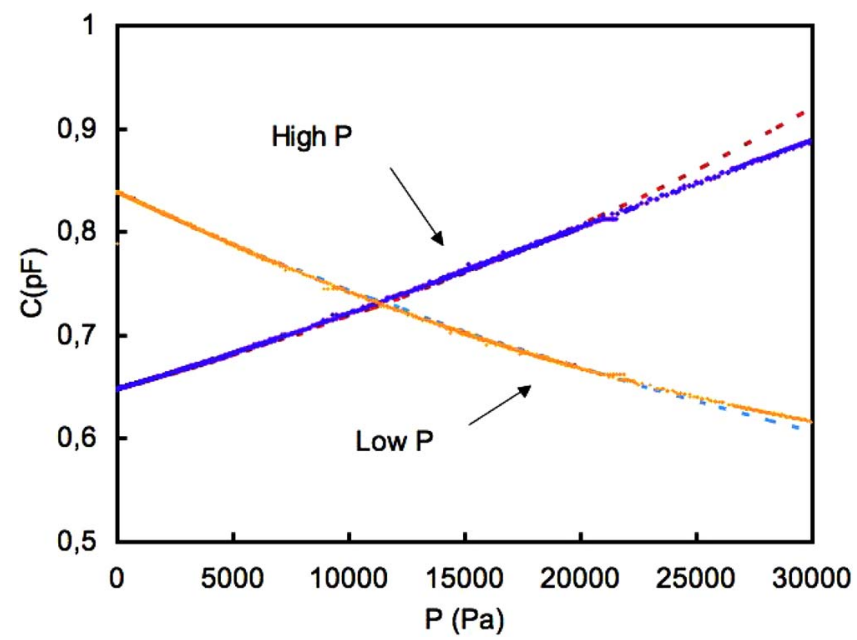

FIG. 4. Calibration at $300 \mathrm{~K}$ of a capacitive pressure cell. The two curves correspond to each side of the cell.

$$
C_{H P}=-\pi \varepsilon_{0} \varepsilon_{R}^{H P} \frac{a^{2}}{l_{0}}\left\{\arctan \left(\frac{R^{2}-r^{2}}{a^{2}}\right)-\arctan \left(\frac{R^{2}}{a^{2}}\right)\right\} .
$$

The dotted lines in Fig. 4 correspond to Eqs. (2) and (3), using $Y=80 \mathrm{GPa}, e=150 \mu \mathrm{m}$, and $\nu=0.3$. For small deflections and for symmetric initial gaps, the capacitance ratio is determined from $X^{-1}=1+\left(\varepsilon_{R}^{L P} / \varepsilon_{R}^{H P}\right)\left(1+2 z / l_{0}\right)$, corresponding to the sensitivity $(\Delta X / X) / \Delta p=-1.5 \times 10^{-5} / \mathrm{Pa}$. For the largest deflections, experiments show that a further correction is necessary, involving an increase of the rigidity due to larger strains on the membrane. However, thanks to a good reproducibility of the results, these data are used as calibration values. Note that a remarkably low level of noise is obtained from the use of a rather rigid membrane associated with a precision capacitance bridge, with a typical resolution better than $0.1 \mathrm{~Pa}$.

This pressure gauge was employed in cryogenic conditions to measure a superfluid helium mass flow rate in a Venturi device. The main difference from the room temperature calibration was an unexplained enhanced threefold sensitivity.

\section{PERIMETER THAT IS WETTED BY A FLUID}

\section{A. Objective}

Many situations require the measurement of liquid levels in both a precise and nonintrusive manner. Capacitive methods use the contrast in dielectric constant between the two phases. This contrast may result from a difference in density of the two phases of a pure body (a liquid and its vapor for instance) or a combination of both the polarizabilities and the densities (a liquid in contact with a gas, two liquid phases of different species). A common configuration for capacitive measurements is the case when the two electrodes constituting the condenser are perfectly coupled. This is achieved with coaxial condensers or parallel plate condensers, with the drawback that the distance between the electrodes is generally less than $1 \mathrm{~mm}$. The reasons for these choices are to obtain large enough capacitances (picofarad or more) and negligible edge effects. For a fluid, the presence of these two closely spaced electrodes may affect its motion (moving fluid) and give rise to unwanted phenomena such as capillary rise.

A geometry of interleaved electrodes deposited on an insulating material, being nonintrusive, has been used by several authors. A version of this system has been patented by Bosch $^{5}$ to measure a fuel film in the intake duct of an internal combustion engine. In that case, several small discrete condensers were used to get information on the film presence at different locations. The Kapton film was glued on a grounded support, and the measurements were performed using a three-terminal method. The patent claim was that this geometry was particularly suitable to screen spurious effects due to electrical coupling, and also to enhance the sensitivity to wetting by the fluid.

This work came to our attention after observing that, while measuring the wetting by a liquid helium film in a pipe with a similar technique, the response was surprisingly high 

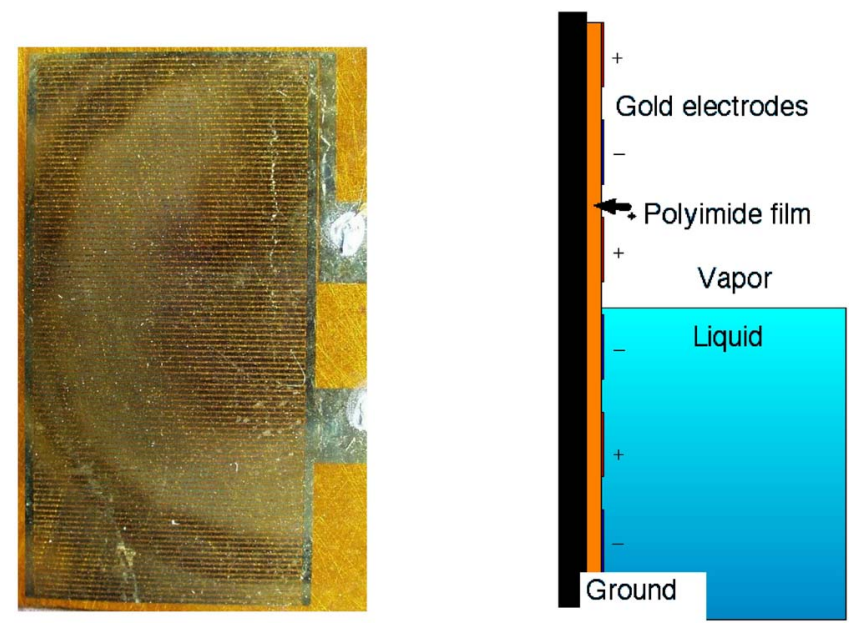

FIG. 5. Left: Photograph of a planar condenser with gold electrodes evaporated on a Kapton film. The two interleaved combs are separated by a $100 \mu \mathrm{m}$ wide gap. Each comb is constituted of 50 teeth. Right: Schematic illustration of the arrangement used for liquid level measurements (in the absence of meniscus).

and reproducible. The reasons for this interest in cryogenic superfluid two-phase flow in a horizontal pipe is discussed in Refs. 6 and 7. The results observed under cryogenic conditions with superfluid helium are not specific to this fluid, and its particularly low dielectric constant (1.056 at saturating vapor pressure and $1.8 \mathrm{~K}$ ) nicely illustrates the high sensitivity that can be obtained with this method.

\section{B. Designing the sensors}

For a continuous liquid level detection, the electrodes forming the planar condenser are evaporated on a flexible plastic film in an interleaved geometry shown in Fig. 5. With $t$ the thickness of the Kapton polyimide, $w$ the width of the electrodes, and $g$ the gap between electrodes, the periodicity of the structure is $\lambda=2(w+g)$. For a typical arrangement on a rectangular $15 \times 30 \mathrm{~mm}$ geometry using $t=50 \mu \mathrm{m}$, $w=200 \mu \mathrm{m}$, and $g=100 \mu \mathrm{m}$, the total length of pairs of opposite electrodes is $L=1.5 \mathrm{~m}$. Figure 5 illustrates this arrangement.

Much effort was devoted to obtaining a metallic layer which is firmly bound to the substrate. After careful cleaning of the Kapton film with reactive ion etching, good adhesion was achieved by the successive evaporation of a $10 \mathrm{~nm}$ thick Ti layer followed by a $200 \mathrm{~nm}$ thick layer of gold. The interleaved pattern is obtained either using a negative resin with directional evaporation on the Kapton, or a positive resin and chemical etching of the gold on a preplated sample. The condenser is eventually glued with epoxy on a metallic substrate in the desired experimental arrangement.

Figure 6 illustrates the case where the condensers were designed to fit inside the inner perimeter of a $40 \mathrm{~mm}$ stainless-steel pipe. A thin metallic $\mathrm{Cu}$-Be ring supports four condensers glued on its inner circumference. A Teflon cylinder was used to apply pressure on the condensers during the gluing process. The $\mathrm{Cu}-\mathrm{Be}$ ring is then inserted in the pipe and the electrodes are electrically connected using $\mathrm{Cu}$ wires

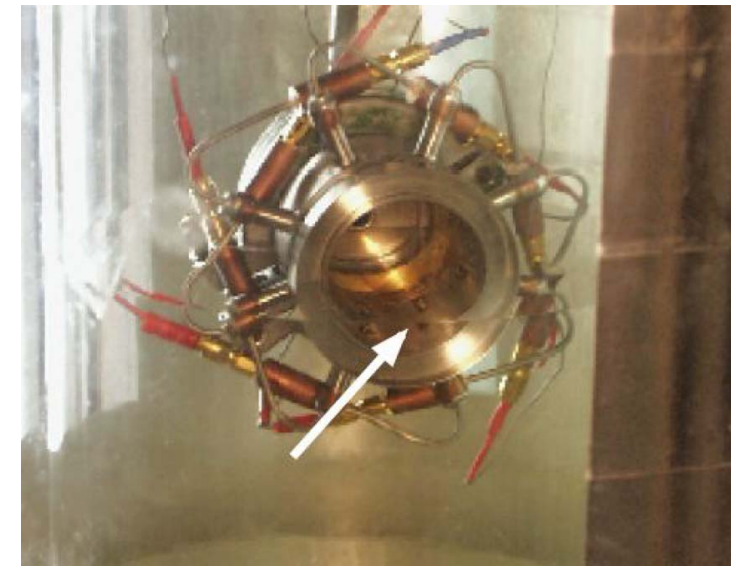

FIG. 6. Photograph of a cylinder containing four planar condensers tested in superfluid helium at saturated vapor pressure $(T=1.8 \mathrm{~K})$. The liquid-gas interface in the Dewar is shown by the arrow.

and silver paint. This geometry was used to measure liquid levels in stratified two-phase flows of helium without perturbation. $^{8}$

\section{Calibrating the sensors}

A major improvement of the system as compared to that patented by Bosch is that the sensitivity to the fluid is tunable by a proper choice of the geometry. The claim of the patent is that, "The capacitive sensor according to the invention has the advantage that the relative capacitance change on wetting the electrodes is large, since the additional ground electrode reduces the capacitive coupling between the electrodes by the rear side of the sensor, so that the largest capacitive contribution is supplied by the space between the electrodes on the one or top sensor side. Furthermore, the ground coating on the sensor rear side (or side remote from the side the electrodes are on) acts as a shield, so that the influence of interfering electrical fields is neutralized and the grounding surfaces in the vicinity of the rear side of the sensor do not influence the characteristic properties of the sensor."

However, many situations require a quantitative knowledge of the sensor response to a given change in the fluid dielectric constant, and this point was not addressed.

To achieve this goal, the response of several interleaved condensers was measured after immersion in fluid of various dielectric constants. The high-precision bridge used for the measurements is commercialized by Andeen-Hagerling ${ }^{9}$ and works in a differential configuration using a built-in reference capacitor. Experimentally, the measured capacitances are compatible with a simple model of two capacitances in parallel given by Eq. (4),

$$
C_{\text {measured }}=C_{\text {ins }}+C_{\text {fluid }} \text {. }
$$

The physical interpretation of $C_{\text {ins }}$ is therefore the capacitance associated with the field in the insulated side (Kapton plus glue, interrupted by the plane at zero potential), and $C_{\text {fluid }}$ is the capacitance of the half-space occupied by the fluid phase. If $C_{\text {vacuum }}$ represents the capacitance in vacuum, $C_{\text {fluid }}$ is given by Eq. (5), and a sensitivity factor $f$ is defined from Eq. (6), that corresponds to the field lines that are affected by the presence of the fluid, 


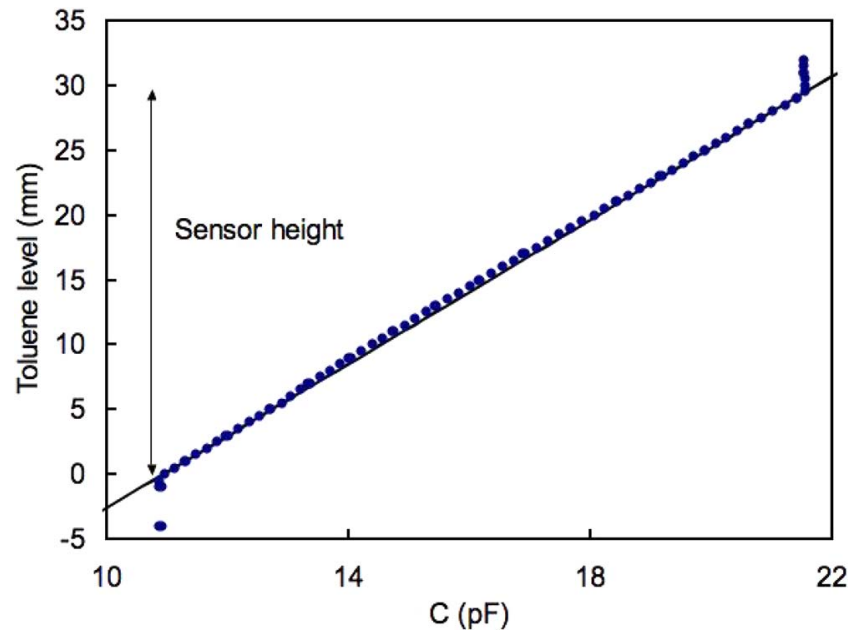

FIG. 7. Test of the linear response of a capacitive sensor progressively immersed in liquid toluene.

$$
\begin{aligned}
& C_{\text {fluid }}=\varepsilon_{R}^{\text {fluid }} \times C_{\text {vacuum }}, \\
& f=\frac{C_{\text {vacuum }}}{C_{\text {ins }}+C_{\text {vacuum }}} .
\end{aligned}
$$

A general expression is obtained with Eq. (7) that is valid for both the gas and the liquid phase, and that contains the factor $f$, which is a characteristic of the condenser,

$$
C_{\text {bridge }}=C_{\text {vacuum }}\left(1+f\left(\varepsilon_{R}^{\text {fluid }}-1\right)\right) \text {. }
$$

It was found that, for several condensers having the same period $\lambda$ and same substrate thickness, and with several fluids at different temperature and for different dielectric constants (air $\left[\varepsilon_{R} \approx 1\right]$, toluene $\left[\varepsilon_{R}=2.38\right]$, liquid nitrogen $\left[\varepsilon_{R}=1.44\right]$, svp helium at $4.2 \mathrm{~K}\left[\varepsilon_{R}^{\mathrm{liq}}=1.049, \varepsilon_{R}^{\mathrm{vap}}=1.005\right]$, svp superfluid helium at $\left.1.8 \mathrm{~K}\left[\varepsilon_{R}^{\mathrm{liq}}=1.0573, \varepsilon_{R}^{\mathrm{vap}}=1.0002\right]\right)$, that the sensitivity factor $f$ was constant at $0.70 \pm 0.03$. This observation suggests that the observed dispersion is due to the nonreproducible process of gluing. It is remarkable that the absolute value of $C_{\text {vacuum }}$ may change by $10 \%$ or more, when for instance some of the teeth of the comb are missing, but this has no consequence on the dynamics of the measurements as defined by the factor $f$.

\section{Linear behavior of the liquid level sensors}

The response of the capacitive sensors as a function of the wetted fraction has been tested in several configurations. Figure 7 shows the capacitance of a vertical rectangular sensor as a function of the immersed depth in toluene. The behavior is linear as expected, and the slope follows Eq. (7). In this experiment, the liquid level is fixed, and the sensor progressively immersed in the liquid. The absolute physical origin is not known, as the response starts with the rise of the meniscus of the fluid on the sensor. No prewetting occurs under these conditions, but the dewetting is partially hysteretic, and was not systematically investigated.

Another test was performed in superfluid liquid helium at saturated vapor pressure and $2 \mathrm{~K}$ in an optical cryostat with the capacitor configuration shown in Fig. 6. In the experiment, the responses of four capacitive sectors are re-

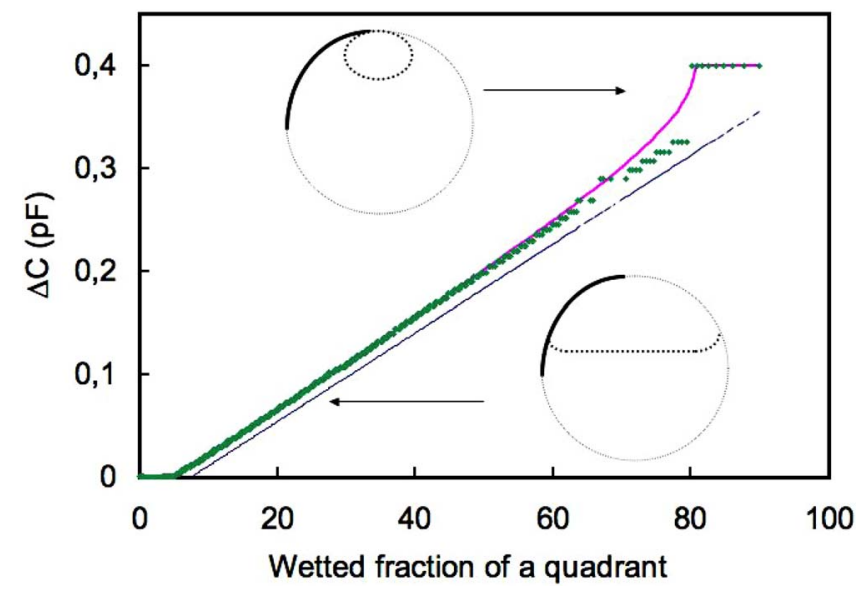

FIG. 8. Calibration curve of one of the two top sensors in superfluid helium. The symbols correspond to experimental data. The dotted line corresponds to the free-surface level and the continuous line accounts for surface tension effects.

corded as the liquid level decreases due to evaporation. The liquid height $h$ is measured optically from the bottom of the cylinder of radius $R$, and Fig. 8 represents the response of the sensor having its end at the top of the cylinder, and its bottom near the midheight of the cylinder. The plot represents the change in capacitance as a function of the quadrant perimeter given by $R / 2 \times \arccos [1(-h / R)]$. The total change in capacitance agrees with previous calibrations, but dewetting is delayed due to the presence of the meniscus. This corresponds to the jump observed on the figure at high liquid level. At midheight of the cylinder, the wall is perpendicular to the liquid-free surface, and the meniscus height given by $\sqrt{2} \lambda_{C}$, where $\lambda_{C}$ is Laplace's length $\left(\lambda_{C}=0.46 \mathrm{~mm}\right.$ for helium at $1.8 \mathrm{~K})$.

\section{E. Thickness of a liquid film}

An analytic 2D solution is known for a structure which is symmetric with respect to the plane of the electrodes, which are of negligible thickness. ${ }^{10}$ In a semi-infinite comblike structure, the electric field must be evanescent in a direction $z$ perpendicular to the plane of the electrodes. Defining a wave vector $k_{0}=2 \pi / \lambda$, with $\lambda$ the periodicity of the potential, one shows that the electric field is localized in the vicinity of the structure, over a typical distance given by $\lambda / 4 \pi$. This result was used in Ref. 11 to measure the thickness and to manipulate thin films of helium on a silicon substrate with an interleaved structure with a gap size of some micrometers. Note that in the present case, the size of the gap being $100 \mu \mathrm{m}$ instead of $5 \mu \mathrm{m}$ in their study, the electrical field near the structure is 20 times less and the electrostatic forces 400 times less for the same polarization of the electrodes (typically $8 \mathrm{~V}$ ).

In order to extend these exact results to the film thickness dependence of interleaved electrodes on Kapton, the problem was solved numerically using the electrostatic finite element module of the ANSYS software. The boundary conditions are defined by the exact geometry of the electrodes, and the electric field normal to the electrodes computed from the charges. An additional insulating layer between the 


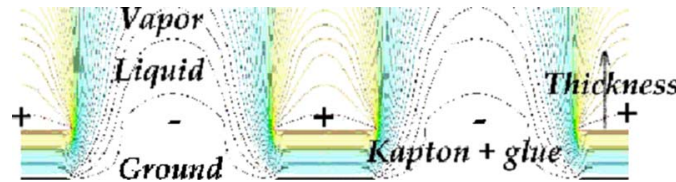

FIG. 9. Example of equipotential lines close to the electrodes for an interleaved structure of gold electrodes on Kapton. The liquid is helium, of relative dielectric constant $\varepsilon_{R}^{\mathrm{liq}}=1.057$ at $1.8 \mathrm{~K}$.

$50 \mu \mathrm{m}$ thick Kapton foil and the ground was considered, corresponding to the epoxy glue. For simplicity, a total thickness of plastic $t=70 \mu \mathrm{m}$ (Kapton plus glue) is used, and the dielectric constant is adjusted to mimic the experimental data. An example of equipotential lines is shown in Fig. 9 in a typical configuration corresponding to wetting by a thin helium film. In this case, the dielectric constant is so close to 1 that the equipotential lines are hardly affected by the fluid presence.

The response of a sensor as a function of the film thickness is given in Fig. 10. The insert on the graph represents the same data on a semilog plot, according to Eq. (8). The capacitance $C_{L_{f l}}$, corresponding to a film of thickness $L_{f l}$, is normalized relative to $C_{\infty}$ corresponding to very thick films and to $C_{0}$ with no liquid on the electrodes,

$$
C_{\infty}-C_{L_{f l}} \propto \exp ^{-2 k_{0} L_{f l}} .
$$

The slope -0.021 (in $\mu \mathrm{m}^{-1}$ ) resulting from the numerical simulation compares favorably with an evanescent field decaying over the typical length $\lambda / 4 \pi=48 \mu \mathrm{m}$. A more careful analysis with high-order terms for the potential shows that the sensitivity is enhanced for small films, and tends to an exponential law for thick films.

\section{F. Discussion}

As expected, an interleaved structure covered by a liquid film of small dielectric constant in the presence of a grounded plane on the opposite side reproduces the proper- ties of the evanescent electric field. This result has been obtained from a comparison with the analytic solution of the 2D symmetrical geometry, and relies on the periodicity of the potential.

However, a direct comparison for the total capacitance obtained from the analytic solution of the 2D geometry and that measured experimentally is notably different. Denoting $K$ the complete elliptic integral of first kind and $K^{\prime}$ the complementary function, one expects a capacitance per unit length and per pair of electrodes, given by Eq. (9),

$$
C=2 \varepsilon_{0} \frac{K\left(\sin k_{0} \frac{a}{2}\right)}{K^{\prime}\left(\sin k_{0} \frac{a}{2}\right)} .
$$

Using the geometric parameters $w=200 \mu \mathrm{m}, g$ $=100 \mu \mathrm{m}$, and the developed length of $L=1.5 \mathrm{~m}$ of paired electrodes gives 34 or $17 \mathrm{pF}$ per half-space. This is more than twice what is measured for the contribution on the side of the fluid, given by $C_{\text {vacuum }}=f \times C_{\text {empty }} \approx 7.7 \mathrm{pF}$. The grounded plane thus decreases the absolute capacitance of the open space.

A simple scheme is proposed to understand the high sensitivity of these sensors when modeled into two separate half-spaces. The value of the capacitance $C_{\text {ins }}$ is minimal, despite a large dielectric constant $\left(\varepsilon_{R}=3.4\right)$, because its thickness is less than $\lambda / 4 \pi$. For the open half-space, the total capacitance is reduced due to the proximity of a grounded plane, but its dynamics is given by the fluid dielectric constant.

A more systematic electrostatic study of the influence of the geometric parameters has also been performed and will be published elsewhere. ${ }^{12}$ It shows that the sensitivity of planar condensers in the adopted geometry is comparable to that of a condenser with perfectly coupled electrodes, but with a reduced capacitance. We obtained for instance on $50 \mu \mathrm{m}$

\section{Csim \\ (pF)}

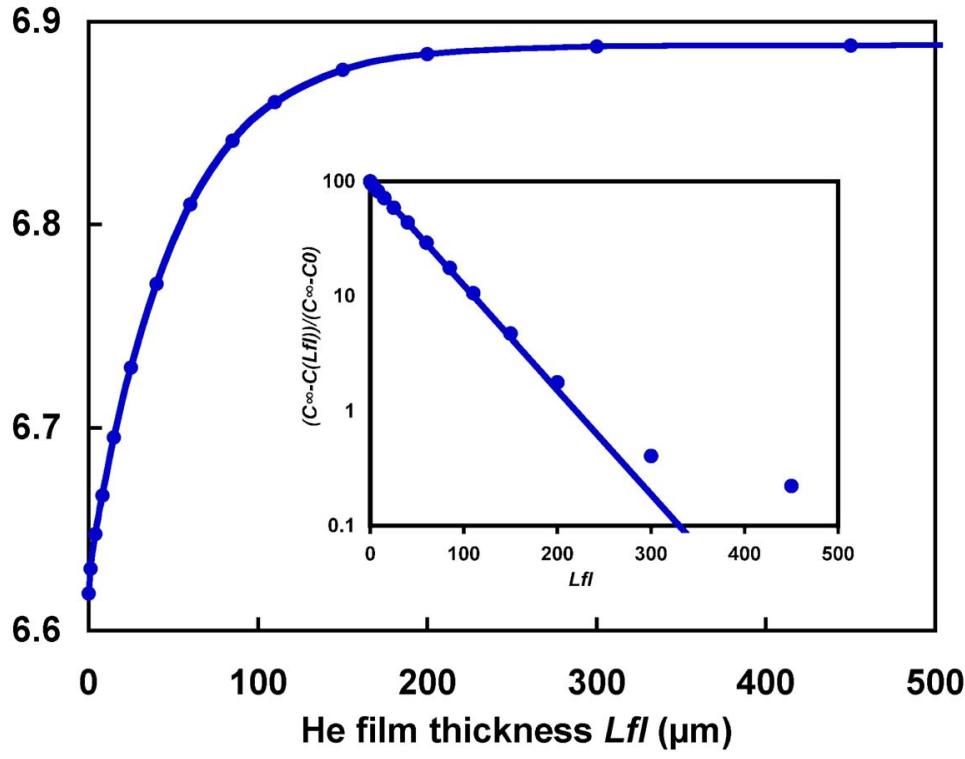

FIG. 10. Numerical simulation of the response of a capacitive sensor to the wetting by a liquid helium film of thickness $L_{f l}$ (gap $g=100 \mu \mathrm{m}$, period $600 \mu \mathrm{m})$. Insert: same data normalized on a semilog scale. 


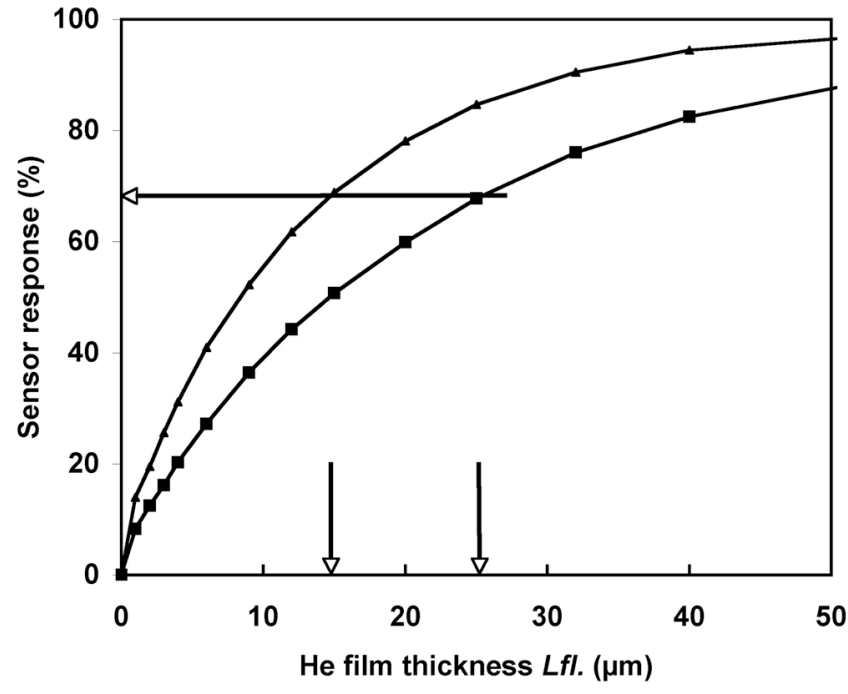

FIG. 11. Comparison of the responses of two condensers of gap $g=30 \mu \mathrm{m}$ (top curve), and $g=50 \mu \mathrm{m}$ (lower curve). See the text for explanations.

thick Kapton substrates experimental sensitivity factors that are, respectively, $f=0.36,0.43,0.70$ in the case of interleaved electrodes presenting gaps $g$ of 30,50 , or $100 \mu \mathrm{m}$ and for electrode widths $w=2 g$.

For practical purposes, in a single experiment, one cannot distinguish between the partial wetting of a sensor by bulk liquid, or by a continuous thin film. Figure 11 illustrates this result in a fictive situation. It shows that a sensor response of $68 \%$ can be obtained in two different ways. Assuming the film is continuous, it corresponds to a $15 \mu \mathrm{m}$ thick film (structure with a gap of $30 \mu \mathrm{m}$ ) or to a $25 \mu \mathrm{m}$ thick film (structure with a gap of $50 \mu \mathrm{m}$ ). Assuming the film is thick with respect to both characteristic lengths, we would deduce that the surface that is wetted by the fluid is $68 \%$ of the total sensor surface on both structure. A combination of sensors of different gap size may thus be used (see Ref. 13, for instance) to distinguish between theses two cases, as the responses are thickness but also coverage dependent.

\section{CONCLUSION}

Many devices working on the principle of capacitive detection using flexible electrodes are discussed in this article. The examples that are given are not exclusive, but cover a number of possible applications. For each use, particular at- tention has to be devoted to the most suitable design to reach the desired sensitivity. For many realizations, minimal effort is required, particularly when the electrodes do not need micron-size resolution. In this case, standard tools for electronic circuitry design are sufficient. Some applications may not require capacitive bridges as accurate and expensive as those cited in this article as long as they include a threeterminal measurement configuration. In particular, we recently obtained good results using the two-channels capacitive-to-digital AD7746 Analog Devices ${ }^{14}$ converter associated with its evaluation board. It allows both differential and absolute capacitive measurements with a typical femtofarad resolution, with a $\pm 4 \mathrm{pF}$ dynamic range for a condenser with initial capacitance value between 0 and $17 \mathrm{pF}$. For this reason, we have designed capacitors corresponding to this dynamic range, and obtained accurate liquid level measurements. The system being very affordable, we plan to generalize its use to other contexts.

\section{ACKNOWLEDGMENTS}

The authors are grateful to C. Lemonias, B. Fernandez, and T. Crozes, for their invaluable help in the realization of the samples, J.-L. Bret for improving the measurements, and C. Guttin for drawing our attention to the performances of the Analog Devices capacitive to numeric converter. The authors would also like to thank J. Butterworth from Air Liquide Co. for his help in preparing this article.

${ }^{1}$ E. D. Adams, Rev. Sci. Instrum. 64, 601 (1993).

${ }^{2}$ P. Thibault, J.-J. Préjean, and L. Puech, Phys. Rev. B 52, 17491 (1995).

${ }^{3}$ ABB Barras-Provence capacitive bridge, 04100 Manosque, France.

${ }^{4}$ S. Timoshenko, Résistance des Matériaux, 2nd ed. (Dunod, Paris, 1968), Vol. 2.

${ }^{5}$ H. Magenau, N. Simon, H.-E. Bollhagen, S. Steinlechner, and B. Wocher, for Bosh GmbH, US Patent No. 5,175,505 (1992).

${ }^{6} \mathrm{Ph}$. Lebrun, Cryogenics 34, ICEC Supplement, 1-8 (1994).

${ }^{7}$ L. Grimaud, A. Gauthier, B. Rousset, and J. M. Delhaye, Cryogenics 37, 739 (1997).

${ }^{8}$ P. Thibault, E. di Muoio, L. Puech, B. Rousset, and P.-E. Wolf, AIP Conf. Proc. 613, 1683 (2002).

${ }^{9}$ Model AH 2550A, Andeen-Hagerling Inc., 31200 Bainbridge Road, Cleveland, OH 44139-2231.

${ }^{10}$ L. Landau and E. M. Lifschitz, Course in Theoretical Physics VIII, Electrodynamics of Continuous Media (Pergamon, Oxford, 1960).

${ }^{11}$ F. Portier, Ph.D. thesis, Université de Paris (2002).

${ }^{12}$ P. Thibault, B. Rousset, R. Vallcorba, and P.-E. Wolf (unpublished).

${ }^{13}$ S. Perraud, Ph.D. thesis, Université Grenoble 1 (2006).

${ }^{14}$ Analog Devices evaluation board, model AD7746, datasheet available from http://www.analog.com 